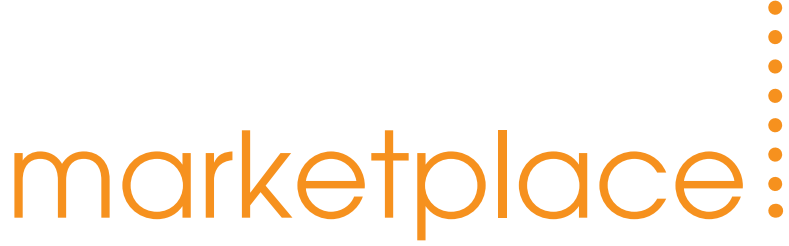

Marketplace is provided as a service to readers using text and images from the manufacturer, supplier or distributor and does not imply endorsement by Vital. Normal and prudent research should be exercised before purchase or use of any product mentioned.

\section{Paste for pulpitis}

Ledermix Dental Paste is an anti-inflammatory and antibiotic treatment that is particularly useful in the emergencymanagement of patients with irreversible pulpitis.

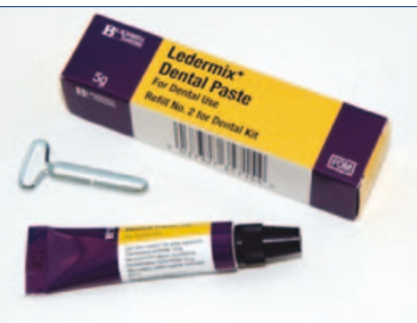

The product gives patients with irreversible pulpitis effective pain relief until definitive root canal treatment can be performed. It is simple to use and versatile and it can be used to fill canals after pulp extirpation during endodontic therapy. After application, the cavity can be closed with a cotton wool pledget and a temporary dressing. Ledermix Dental Paste has been designed to be water-soluble so it can be easily rinsed out of root canals.

When canal preparation has been completed, obturation is then performed using your preferred material. It is available from Blackwell Supplies.

\section{Fluoride releasing restorative}

Riva Silver from SDI is a mercury free fluoride releasing glass ionomer silver restorative which has strength, chemical adhesion, fluoride release and easy handling.

Riva Silver releases high levels of fluoride, resulting in increased anticariogenic properties and improved longevity of the restoration. Its condensable nature allows the operator to quickly and easily place the restoration and can be packed using an amalgam condenser. Its high compressive strength minimises the risk of fracture in the critical early stages after the restoration has been placed while the heat accelerated setting mechanism allows the material to 'snap set'. The addition of metal particles into the cement substan-

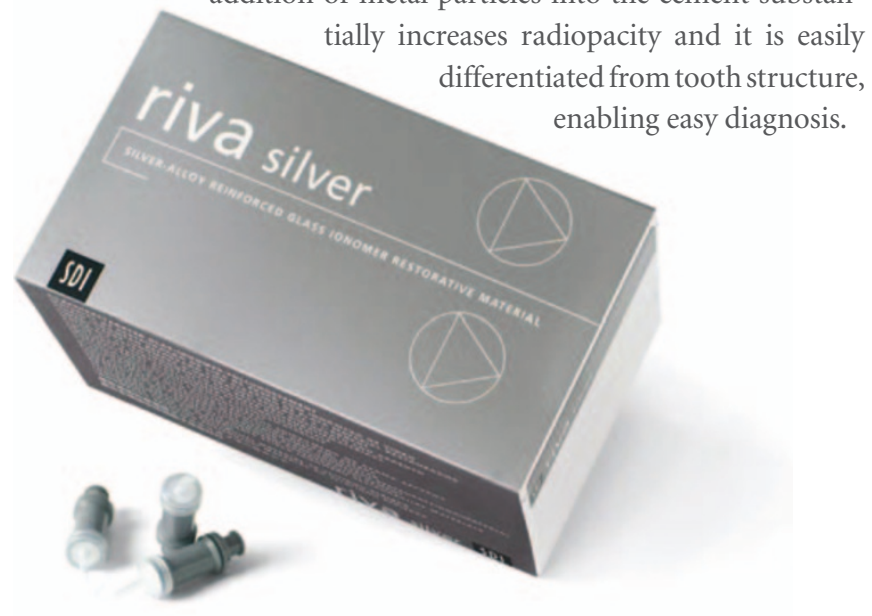

\section{Have your cake and eat it}

There are now a large number of sugar alternatives available to those wanting to avoid sugar, but a new product launched under the brand name Perfect Sweet stands out for its dental benefits, according to its creators.

Perfect Sweet's sole ingredient is xylitol, a name derived from the Greek word 'xyl', meaning wood, as it was first discovered in birch wood. Xylitol is a natural substance found in many plants and fruits, and despite looking and tasting just like sugar it has several healthy benefits. It has nearly half as few calories as sugar, 75\% less available carbohydrates, and a GI (Glycaemic Index) value of just seven, making it ideal for dieters, diabetics, and those looking to eat more healthily.

While sugar forms an acidic environment most other sweeteners either cause a small rise in acidity or don't have an effect on $\mathrm{pH}$ at all, therefore whilst they may not cause damage to teeth, they do not actively help teeth. Xylitol forms an alkaline environment when it is

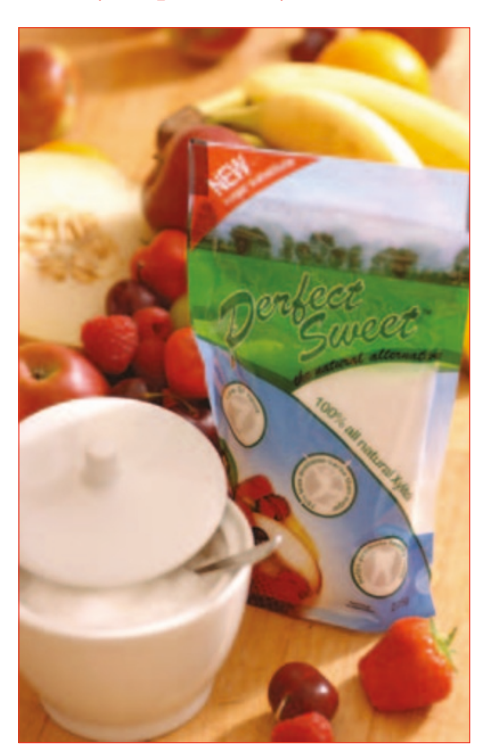

broken down. Studies published on xylitol show that this has two dental benefits. Firstly it means Streptococcus mutans numbers are reduced and secondly it encourages the uptake of the minerals that are needed to repair damaged enamel.

Perfect Sweet is a mainstream xylitol sugar alternative and can be found in Sainsbury's, Waitrose, Tesco, Holland and Barrett, and independent health stores.

For further information and a list of studies visit www. perfectsweet.co.uk.

\section{A wonder hoe}

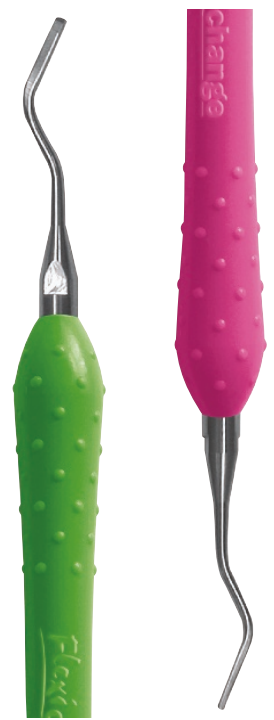

For effective periodontal therapy, it is essential to leave the pocket as clean as possible, but this can be complex with a really deep pocket. The Dentsply Ash WonderHoe has been designed with a longer reach than the average hoe and a slightly extended curvature.

The longer reach and the slightly curved tip means the WonderHoe follows the contour of the root, allowing the instrument to gain deeper access.

All the instruments have Tungsten Carbide tips so they stay sharper for longer and they can also prove very useful for light calculus removal on exposed root surfaces, when an ultrasonic scaler is uncomfortable for the patient. 